\title{
Heart rate turbulence in healthy dogs and dogs with dilated cardiomyopathy
}

\author{
A. Noszczyk-Nowak
}

Department of Internal Disease with Clinic of Horses, Dogs and Cats, Faculty of Veterinary Medicine, University of Environmental and Life Sciences, pl. Grunwaldzki 47, 50-366 Wrocław, Poland

\begin{abstract}
Heart rate turbulence (HRT) is modulated by the baroreceptor reflex and it was suggested that it could be used as a measure of autonomic dysfunction. Impaired HRT is of a significant prognostic value in humans after myocardial infarction, suffering from dilated cardiomyopathy and patients with heart failure. So far no studies were performed assessing the importance of HRT in dogs. The aim of this study was to prospectively evaluate the HRT turbulence onset (TO) and the turbulence slope (TS) in healthy dogs and in dogs with DCM and to compare the HRT in dogs with DCM that died during the first 30 days of observation and dogs with DCM that survived the first 30 days after the HRT analysis. The current study was aimed at determining reference value of the TO and TS of HRT in healthy dogs (control group) and dogs with dilated cardiomyopathy (DCM group). The tests were carried out on 30 healthy dogs and 30 dogs with DCM composed of Boxers, Doberman pinschers and Great Danes, of different sexes and body weights from 22 to $72 \mathrm{~kg}$, aged between 1.5 and 11.5 years, submitted to the 24-hour Holter monitoring. HRT parameters were calculated using an HolCard software algorithm. TO is a percentage difference between the heart rate immediately following ventricular premature complex (VPC) and the heart rate immediately preceding VPC. TS corresponds to the steepest slope of the linear regression line for each sequence of five consecutive normal intervals in the local tachogram. The average TO in healthy dogs was determined as $-13.55 \pm 11.12 \%$, TS was $21.33 \pm 9.66 \mathrm{~ms} / \mathrm{RR}$. TO in dogs with DCM was determined as $-2.61 \pm 2.1 \%$ and TS was 6.15 $\pm 3.86 \mathrm{~ms} / \mathrm{RR}$. Parameters of HRT were statistically significantly decreased $(\mathrm{p}<0.01)$ in dogs with DCM. HRT TO and TS were statistically significantly decreased in dogs with DCM. Dogs with DCM that survived more than 30 days of observation had HRT statistically significantly decreased in comparison to dogs with DCM that died after the 30'th day of observation. Decreased HRT parameters in dogs with DCM suggest an autonomic neuropathy which principally consists of the withdrawal of the cardiac parasympathetic tone. The more the autonomic neuropathy is advanced the faster the death of the dog with DCM might occur, with no correlation with the level of the heart failure.
\end{abstract}

Key words: dogs, dilated cardiomyopathy, heart rate turbulence, baroreceptor reflex. 


\section{Introduction}

The physiological phenomenon known as the heart rate turbulence (HRT) was described by Schmidt et al. in 1999 (Schmidt et al. 1999). HRT describes short term variations in the heart rate after ventricular premature complex (VPC). Shortly after VPC an early heart rate acceleration can be observed followed by the late deceleration. The HRT phenomenon can be observed during 15-20 following cardiac cycles. Heart rate turbulence is quantified by two parameters: the turbulence onset (TO) and the turbulence slope (TS). The mechanism of HRT has not been clearly explained however it is suspected that it is correlated with the baroreflex (Schmidt et al. 1999, Bauer and Schmidt 2003). Baroreceptors located in the aorta's arch and carotid bulb are responsible for one of the most basic mechanisms controlling arterial pressure and the heart rhythm. Baroreceptors react faster at sudden drops of the arterial pressure than at its increase so they are constantly stimulated by changes of the arterial pressure. Ventricular excitations evoke rapid, transient drop of arterial pressure. This evokes the baroreceptors' reaction leading to parasympathetic system activity reduction. A consecutive domination of the sympathetic system and the decrease of the parasympathetic system's tone leads to the increase in the heart rate, following the reaction of the barorecptors. The increase of the arterial pressure after VPC leads to an opposite reaction - activation of the parasympathetic system and a reduction of the heart rate (Bauer and Schmidt 2003). The length of the heart cycle changes rapidly after acetylocholine administration as compared to noradrenaline administration. Due to that the dynamic reaction of the sinus node to the rapid changes of the blood pressure is due to the activation of the vagus nerve. Studies performed by Marine et al. show that HRT generally depends on the parasympathetic system as both HRT parameters, TO and TS, are reduced after the administration of atropine (Marine et al. 2002). It is believed that abnormal HRT parameters may mirror lack of the protective anti-arrhythmic performance of the vagus nerve. The evaluation of HRT is used in human medicine to identify people with higher mortality risk due to cardiac diseases. It has been proved that a limitation or lack of HRT in patients after heart infarct is related to a higher risk of death during subsequent observation (Barthel el al. 2002, Sade el al. 2003, Berkowitsch el al. 2004). The suitability of this parameter to evaluate the mortality risk in patients with chronic heart failure $(\mathrm{CHF})$, dilated cardiomyopathy (DCM) and patients with aorta stenosis (AS) has been stated (Koyama et al. 2002, Malberg et al. 2003, Arslan et al. 2008). There are no studies evaluating HRT in healthy dogs and its suitability as a risk factor of a sudden cardiac death in dogs with cardiac diseases

The aim of this study was to prospectively evaluate the HRT TO and TS in healthy dogs and in dogs with DCM and to compare the HRT in dogs with DCM that died during the first 30 days of observation and dogs with DCM that survived the first 30 days after the HRT analysis.

\section{Material and Methods}

\section{Reference population}

The study was performed on 60 dogs divided into two groups.

The control group consisted of 30 healthy dogs of three breeds (10 Boxers, 10 Doberman pinschers, 10 Great Dane dogs) with VPC below 100 observed in a 24-hour Holter monitoring which is indicated as a standard procedure in healthy dogs (Noszczyk-Nowak et al. 2009, Wess et al. 2010). The body weights were between 25 and $72 \mathrm{~kg}$, age from 1.7 to 11.5 years. All dogs did not show any abnormalities in clinical examination, ECG and echocardiography.

The DCM group was composed of 30 dogs with DCM from the same three breeds as the control group (10 Boxers, 10 Doberman pinschers, 10 Great Danes) with a present sinus rhythm and VPC (376-11234 VPC/24h) observed in a 24-hour Holter monitoring. The blood analysis, ECG, echocardiography examination and Holter monitoring were performed during the first visit in the Cardiology Room of the Department of Internal Diseases with Clinic for Horses, Dogs and Cats, Faculty of Veterinary Medicine, Wrocław University of Environmental and Life Sciences. Dogs with DCM were not treated before Holter monitoring. The body weights were between 22 and $71 \mathrm{~kg}$, age from 1.5 to 9 years. All dogs in this group had clinical sings of heart failure (Ib, II and IIIa, ISACHC score) (Freeman et al 2005). Heart failure therapy was commenced immediately after the tests, including Holter examination, had been completed.

\section{Methods}

The animals were qualified based upon earlier investigation, preliminary clinical examination and morphological and biochemical blood tests: alanine aminotransrerase (AST), asparate aminotransferase (ALT), level of urea, creatinine, level of ions $\left(\mathrm{Na}^{+}\right.$, $\mathrm{K}^{+}$, total $\left.\mathrm{Ca}, \mathrm{Mg}^{2+}, \mathrm{Cl}^{-}\right)$. Morphological blood tests 
were performed on an Animal Blood Center abc VET analyzer (ABX Vetpack). Biochemistry tests were performed on MaxMat Pl analyzer (Montpellier). No deviations from normal parameters were detected. All dogs were examined echocardiographically. Standard transthoracic views in M-mode were used to obtain left ventricular ejection fraction (EF) and shortening fraction (SF), left ventricular internal end-diastolic diameter (LVIDd), left ventricular internal systolic diameter (LVIDs), intraventricular septal diameter (IVSd) and left ventricular posterior wall (LVPWd) in diastole and intraventricular septal diameter (IVSs) and left ventricular posteriori wall (LVPWs) in systole. The correction of LVIDd for body weight was achieved by dividing LVIDd $(\mathrm{cm})$ by body weight $(\mathrm{kg})$. The echocardiography examination was performed on the echocardiograph ALOKA 4000+ (Aloka). The probe used for echocardiography was sector type $5 \mathrm{MHz}$ and $7.5 \mathrm{MHz}$. All dogs underwent ECG in the right lateral position on BTL SD08 equipped with net filter and different frequencies of muscular filters.

\section{Holter monitoring}

Holter monitoring was performed on Aspekt 702 (Aspel) and analyzed by a computer software HolCard (Aspel). Single use, self-adhesive electrodes were glued to a shaved and degreased skin of the thorax. The unit was stabilized and secured by a band and a special vest. This way of fixing the unit did allow the animals to move around unrestricted and change positions during sleep and other activities. The dogs managed normal, $24 \mathrm{~h}$ life activities. The owner registered the time of resting, sleep and any other disturbing activities. Before analyzing the data the HRT parameters were manually preprocessed. HRT parameters were calculated using an algorithm from HolCard software. TO which is a measure of the expected normal early sinus acceleration after a VPC and TS which is a measure of late sinus deceleration after VPC are the two components of HRT. TO is the percentage difference between the heart rate immediately following VPC and the heart rate immediately preceding VPC. It is calculated using the equation: TO $=((\mathrm{RR} 1+\mathrm{RR} 2)-(\mathrm{RR}-2+\mathrm{RR}-1)) /(\mathrm{RR}-2$ + RR-1) $* 100 \%$, with RR-2 and RR-1 being the first two normal intervals preceding the VPC and RR1 and RR2 the first two normal intervals following the VPC. TS corresponds to the steepest slope of the linear regression line for each sequence of five consecutive normal intervals in the local tachogram. The TO and TS values were calculated for each single premature ventricle excitation, classified as premature ventricle acceleration (minimum 20\% earlier than predominant rhythm) and afterwards the average values of TO and TS were calculated for each patient. All of the examined dogs had a sufficient number of premature ventricle accelerations during 24 hours, qualifying them for HRT analysis (min. $5 \mathrm{VPC} / 24 \mathrm{~h}$, min. 20 sinus beats after VPC). The HRT analysis was performed for all VPC in patients that were qualified. The differences between the control group and the DCM groups and between dogs that did not survive longer than 30 days of observation and dogs with DCM that died after 30'th day of observation, based on parametric data with standard distribution, were analyzed using t-Student test. In case of non-parametric data or lack of standard distribution U Mann-Whitney test was used. The correlation was analyzed using R Spearman test. The testing was done based on the significance level $\mathrm{p} \leq 0.05$. The statistical analysis was performed with a program STATISTICA, version 7.1.

The studies were approved by the 2nd Local Ethical Commission, permit No. 06/2008.

\section{Results}

The clinical findings in both groups are presented in Table 1. The group of healthy dogs and the ones with DCM showed significantly different TO and TS values (Table 1). Dogs with DCM had significantly lower values of TO and TS. During the day all dogs had negative values. In single cases TO was positive during the night, but the average value of TO for all VPC was negative. Additionally, dogs with DCM had lower HRT parameters compared to healthy dogs corresponding to lack of, or a limitation of, typical physiological changes in the heart rate after VPC (Table 1, Fig. 1, 2). Seven of the dogs with DCM (3 Doberman pinchers, 2 Boxers, and 2 Great Dane dogs) that died during first 30 days after the Holter monitoring had TO not higher than $1 \%$ and TS was below $5 \mathrm{~ms} / \mathrm{RR}$ (Table 2). Three dogs were euthanized on the owners request due to the heart failure progression. These dogs had non-significantly lower TO and TS values compared to dogs that survived the 6 month observation period (Table 2). These values showed statistically significant difference due to the number of dogs that were euthanized. TO did not exceeded 5\% in dogs with DCM that survived the 6 month observation period and dogs that were euthanized and TS was not more than $10 \mathrm{~mm} / \mathrm{RR}$. These parameters were significantly higher in dogs that suddenly died $(\mathrm{p}<0.05)$, but significantly lower than in healthy dogs $(\mathrm{p}<0.01)$

There were no correlations between SF, LVIDd, VPC/24h and HRT TO,TS. 
Table 1. Clinical characteristics of the control group and the DCM group.

\begin{tabular}{|c|c|c|c|}
\hline Parameters & $\begin{array}{l}\text { Control } \\
(n=30)\end{array}$ & $\begin{array}{l}\text { Group DCM group } \\
(\mathrm{n}=30)\end{array}$ & p-value \\
\hline Age (year) & $6.11 \pm 4.43$ & $6.64 \pm 2.36$ & n.s. \\
\hline Body mass (kg) & $44.6 \pm 18.9$ & $39 \pm 22.34$ & n.s. \\
\hline Male sex & $16(53 \%)$ & $19(63 \%)$ & \\
\hline Ejection fraction $(\%)$ & $56.25 \pm 14.31$ & $27.02 \pm 6.7$ & $\mathrm{p}<0.01$ \\
\hline Shortening fraction $(\%)$ & $29.26 \pm 15.12$ & $16.78 \pm 7.61$ & $\mathrm{p}<0.05$ \\
\hline LA/Ao & $1.38 \pm 0.45$ & $2.51 \pm 1.02$ & $\mathrm{p}<0.01$ \\
\hline LVIDd (cm) & $4.85 \pm 1.62$ & $5.9 \pm 1.34$ & $\mathrm{p}<0.01$ \\
\hline LVIDd/body mass & $0.10 \pm 0.02$ & $0.15 \pm 0.09$ & $\mathrm{p}<0.01$ \\
\hline LVIDs $(\mathrm{cm})$ & $4.1 \pm 0.36$ & $4.8 \pm 1.26$ & $\mathrm{p}<0.05$ \\
\hline $\operatorname{IVSd}(\mathrm{cm})$ & $1.08 \pm 0.14$ & $0.98 \pm 0.19$ & ns \\
\hline IVSs $(\mathrm{cm})$ & $1.43 \pm 0.28$ & $1.21 \pm 0.26$ & $\mathrm{p}<0.05$ \\
\hline LVPWd (cm) & $1.06 \pm 0.09$ & $1.01 \pm 0.1$ & n.s \\
\hline LVPWs $(\mathrm{cm})$ & $1.47 \pm 0.29$ & $1.21 \pm 0.23$ & $\mathrm{p}<0.05$ \\
\hline Turbulence onset (\%) & $-13.55 \pm 11.12$ & $-2.61 \pm 2.1$ & $\mathrm{p}<0.01$ \\
\hline Turbulence slope (ms/RR) & $21.33 \pm 9.66$ & $6.15 \pm 3.86$ & $\mathrm{p}<0.01$ \\
\hline $\mathrm{P}$ time $(\mathrm{s})$ & $0.04 \pm 0.003$ & $0.05 \pm 0.007$ & $\mathrm{p}<0.01$ \\
\hline $\mathrm{P}$ amp (mV) & $0.17 \pm 0.05$ & $0.19 \pm 0.08$ & n.s. \\
\hline PQ (s) & $0.1 \pm 0.01$ & $0.11 \pm 0.02$ & n.s. \\
\hline $\mathrm{R}$ amp (mV) & $1.32 \pm 0.43$ & $1.99 \pm 1.1$ & $\mathrm{p}<0.01$ \\
\hline QRS (s) & $0.05 \pm 0.008$ & $0.07 \pm 0.12$ & $\mathrm{p}<0.05$ \\
\hline QT (s) & $0.19 \pm 0.03$ & $0.21 \pm 0.02$ & n.s. \\
\hline HR & $119.43 \pm 22.61$ & $148.26 \pm 26.24$ & $\mathrm{p}<0.05$ \\
\hline $\mathrm{VPC} / 24 \mathrm{~h}$ & $24.6 \pm 18.7$ & $1716 \pm 2828.6$ & $\mathrm{p}<0.01$ \\
\hline
\end{tabular}

Table 2. HRT in dogs with DCM which did not survive over 30 days of observation time and dogs with DCM that died after the 30 'th day of observation.

\begin{tabular}{lcccc}
\hline \multicolumn{1}{c}{ Parameters } & DCM death & DCM euthanasia & DCM live & \\
$\mathrm{n}=7$ & $-0.41 \pm 0.18^{*}$ & $3.01 \pm 0.46$ & $-3.26 \pm 2.26^{*}$ & $\mathrm{p}$-value \\
\hline Turbulence onset $(\%)$ & $2.1 \pm 1.81^{*}$ & $5.7 \pm 0.98$ & $6.81 \pm 2.11^{*}$ & $\mathrm{p}<0.05$ \\
Turbulence slope (ms/RR) & $29.02 \pm 3.8$ & $26 \pm 6.6$ & $27.12 \pm 9.8$ & $\mathrm{p}<0.05$ \\
Ejection fraction $(\%)$ & $18.17 \pm 5.65$ & $15.31 \pm 4.34$ & $16.68 \pm 9.95$ & n.s. \\
Shortening fraction $(\%)$ & $6.02 \pm 1.18$ & $6.06 \pm 0.96$ & $5.94 \pm 1.54$ & n.s. \\
LVIDd $(\mathrm{cm})$ & & & n.s. \\
\hline
\end{tabular}

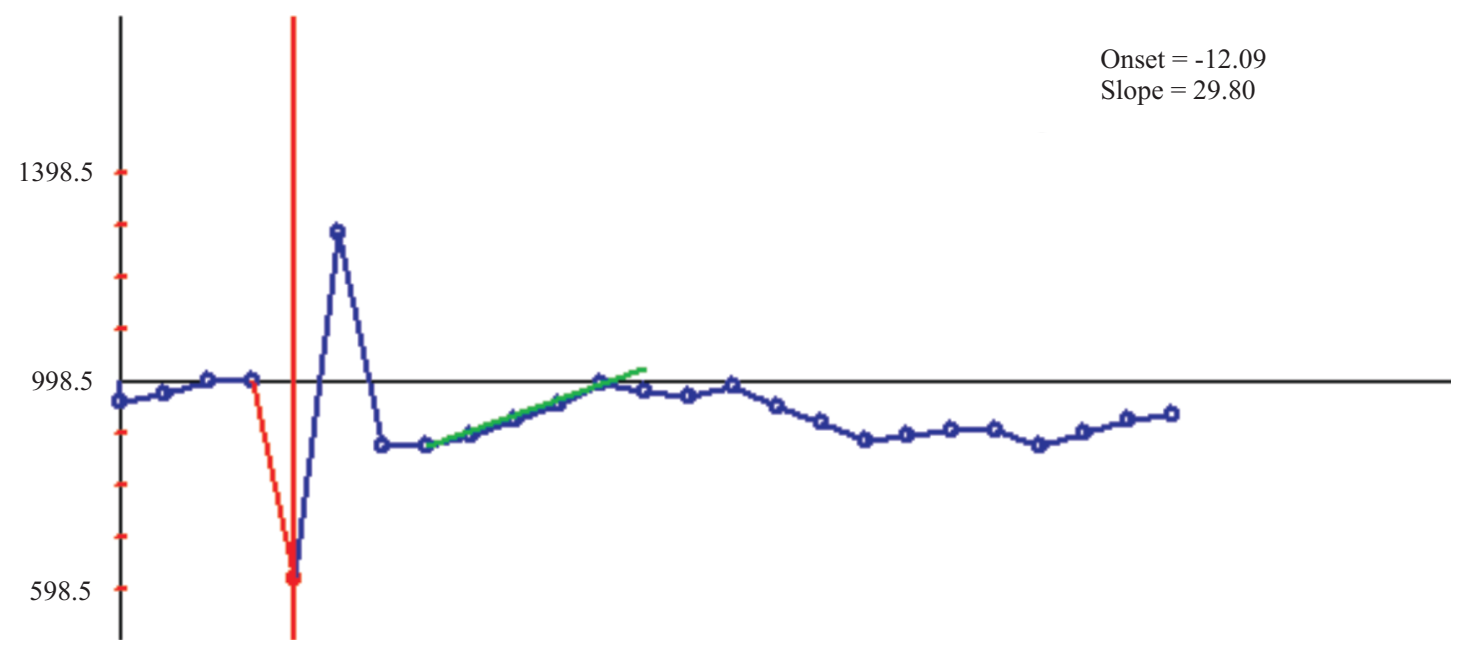

Fig. 1. Healthy dog tachogram. 


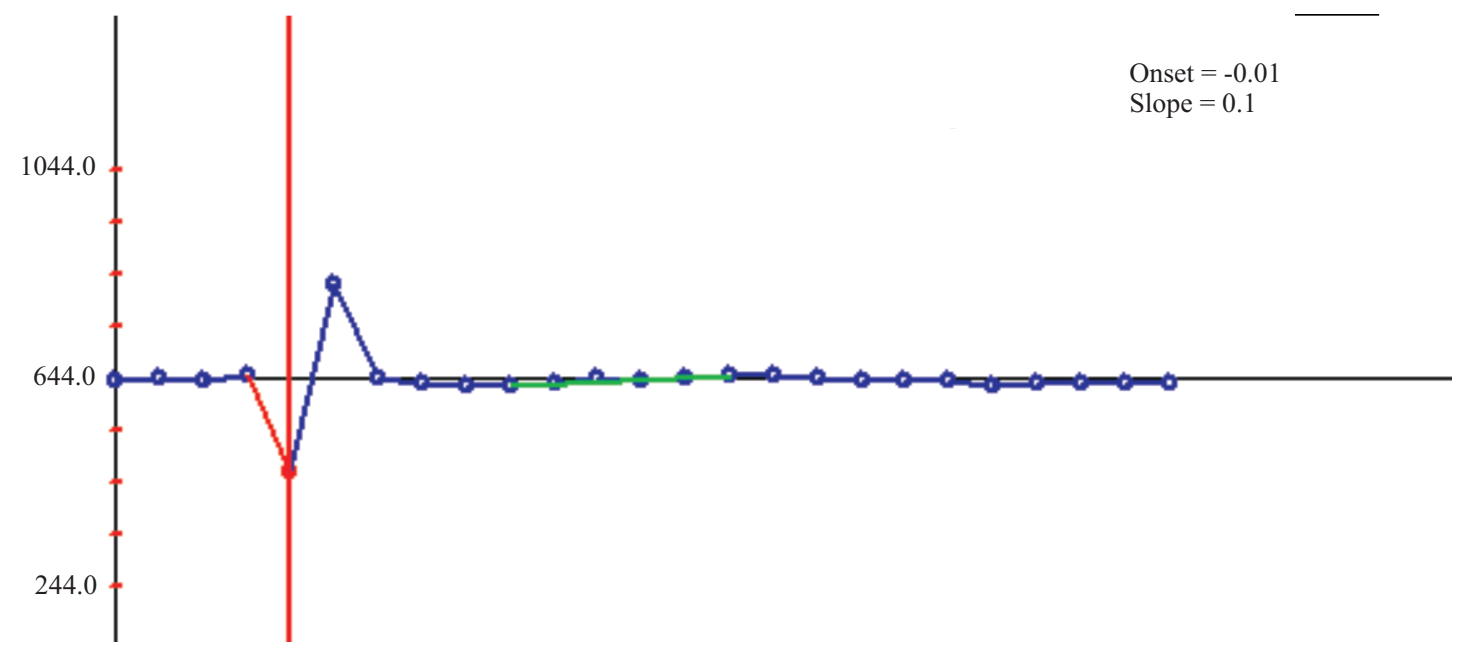

Fig. 2. Tachogram from a dog with DCM which did not survive over 30 days of observation time.

\section{Discussion}

Dilated cardiomyopathy (DCM) is one of the most common organic heart failures observed in dogs, especially in Boxers, Doberman pinschers and Great Danes (Oyama et al. 2009, Wess et al. 2010). Some breeds show DCM predisposition correlated with sex - males seem to suffer from DCM more often than females, what can be seen in the examined group of dogs with DCM (Wess et al. 2010). Dogs with DCM have a smaller shortening fraction and left ventricle ejection fraction. They also show a significant enlargement of the heart chambers which is related to the DCM pathophysiology. Dogs with DCM have an above-average heart rate which is related to an increased adrenergic leading, withdrawal of the parasympathetic tone and a decline in the circadian variability (Calvert et al. 2001). During the first 30 days of observation, in spite of the treatment (pimobendan, beta-blocker, ACE- inhibitor), 7 dogs died (3 Dobermans, 2 Great Danes and 2 Boxers). It was a sudden death during sleep or exertion which suggests death due to arrhythmia. Rest of the dogs with DCM showed heart failure progression leading to the euthanasia of the next three dogs during the 6 month observation period. During the same time one of the dogs from the control group died in a traffic accident. Dogs with DCM had significantly lower values of TO and TS compared to healthy dogs. These results confirm the disorders of the cardiovascular regulation in dogs with DCM. Based on experimental research and clinical tests it is confirmed that there are difficulties to accomplish stable cardiovascular parameters e.g. heart rate after parasympathetic blockade with atropine, blood pressure and heart rate by intravenous injections of phenylephrine in heart failure. This suggests severe reflex and long-term disorders of the cardiovascular regulation system (autonomic neuropathy) (Eckberg et al. 1971, Kubo et al. 1990, Buccafusco 1996, Little et al. 2005). In 1971 Eckberg et al. described a weaker control of the heart rate frequency related to the baroreflex in patients with congestive heart failure (Eckberg et al. 1971). Eaton et al. described changes in the heart rate variability (HRV) parameters in dogs with experimental heart failure. Suggesting a decreased parasympathetic modulation (Eaton et al. 1995). Similar results were obtained by Haggstrom et al. in Cavalier King Charles Spaniels with severe mitral regurgitation (Haggstrom et al. 1996). Autonomic neuropathy in the chronic heart failure is a condition which reduces the baroreflex sensitivity and increases the adrenergic tone. Recently published papers indicate that excessive activation of the beta-adrenergic receptors in the brain is a result of the heart failure but also its reason (Leenen 2007, Gourine et al. 2008). Additionally, a progressive inflammation process in the central nervous system induced by the myocardium damage leads to the dysfunction of the parasympathetic neurons and a decrease of the parasympathetic tonus (Biel et al. 2007). The performed studies confirmed a strong correlation between the HRT decline and the dog's mortality rate (dogs with DCM) in a short period of time after diagnosing HRT disorders. The results obtained from dogs that survived the 6 month observation period suggest that the risk of sudden cardiac death in dogs with DCM is lower if the HRT parameters show that there is a minor disfunction of the barorecptor reaction. The evaluation of the baroreflex (BRS) is difficult and sometimes even impossible to perform in a standard veterinary clinic, however, could bring many useful information regarding the mortality risk of dogs with heart failure. Very promising results from non-invasive, real-time measurements of cardiac vagal 
tone in dogs with cardiac disease were obtained by Little et al (Little et al. 2005). However, this method demands a special electronic device (NeuroScope). The HRT evaluation, related to the baroreflex, in dogs is relatively easier to perform (Bauer and Schmidt 2003). It is important to correctly classify the premature beat, as HRT does not appear after supravetricular accelerations, intercalated accelerations and surrogate ventricular accelerations. It is suggested that the heart rate is controlled by the direct impact of the vagus nerve on the activity of the sinus node and the conduction velocity in the atrioventricular node (Malik et al. 1999). The latency time of the heart's reaction to the vagus nerve activation is very short, about $240-450 \mathrm{~ms}$ in humans and $160-180 \mathrm{~ms}$ in dogs (Little et al. 1999, Jurek et al. 2007). It means that the heart reacts immediately to each single change of the vagus nerve activity as a response to the baroreflex. Neurons in the nucleus tractus solitaris (NTS) are responsible for the overriding control of the arterial pressure. They receive information from the baroreceptors via afferent way. NTS connects different brain regions containing cardiovascular neurons and thanks to this NTS becomes the integration area adapting the circulatory system to functions of other organs (Lawrence and Jarrot 1996). During simultaneous recording of the atrial pressure's wave and the heart rate in healthy people and patients with heart failure it was noticed that during VPC and shortly after there is an immediate decrease of the systolic and diastolic blood pressure (Schmidt et al. 1999, Bauer and Schmidt 2003). However, patients with functionali disorders of baroreceptors do not show specific changes in the heart rate, such as HRT. Similar reactions were observed in dogs with DCM. The TO and TS values were significantly lower which leads to little or lack of changes in the heart rate after VPC. It is particularly observed in dogs with DCM that died suddenly and the TO did not overcome $1 \%$ while TS was $5 \mathrm{~mm} / \mathrm{RR}$. This shows the lack of the physiological heart rate turbulence. The European Cardiology Association stated that HRT is the measure of the vagus nerve activity. During the retrospective analysis it was confirmed that HRT is an independent, specific mortality predictor in humans (Malik et al. 1999).

\section{Study limitations}

The HRT evaluation was performed only in Boxers, Doberman pinchers and Great Dane dogs with a present sinus rhythm and it is not certain that the findings are applicable to other breeds. It is impossible to apply this method in dogs with supraven- tricular dysrhythmias such as atrial flutter or fibrillation. Minimum 5 VPC must be present to evaluate HRT. During the study only the dogs with heart failure due to DCM were examined. Holter monitoring is an expensive examination and the HRT analysis is time-consuming.

\section{Conclusions}

TO and TS HRT were statistically significantly decreased in dogs with DCM. HRT in dogs with DCM which did not survive over 30 days of observation time is statistically significantly decreased in comparison to dogs with DCM that died after the 30'th day of observation. Dogs with DCM with decreased HRT parameters have an autonomic neuropathy which principally consists of the withdrawal of the cardiac parasympathetic tone. The more the autonomic neuropathy is advanced the faster the death of the dog with DCM might occur, with no correlation with the level of the heart failure

\section{Acknowledgments}

This work was supported by the Polish Ministry of Science and Higher Education (Grant No. N N308 071037).

\section{References}

Arslan U, Ozdemir M, Kocaman SA, Balcioglu S, Cemri M, Cengel A (2008) Heart rate variability and heart rate turbulence in mild-to-moderate aortic stenosis. Europace 10: $1434-1441$

Barthel P, Schneider R, Bauer A, Ulm K, Schmitt C, Schömig A, Schmidt G (2003) Risk stratification after acute myocardial infarction by heart rate turbulence. Circulation 108: 1221-1226.

Bauer A, Schmidt G (2003) Heart rate turbulence. J Electrocardiol 36: 89-93.

Berkowitsch A, Zareba W, Neumann T, Erdogan A, Nitt SM, Moss AJ, Pitschner HF (2004) Risk stratification using heart rate turbulence and ventricular arrhythmia in MADIT II: usefulness and limitations of a 10-minute holter recording. Ann Noninvasive Electrocardiol 9: 270-279 .

Biel B, Jankowska EA, Banasiak W, Ponikowski P (2007) The effect of cholinergic stimulation on autonomic balance and immune response in patients with chronic heart failure. Kardiologia Polska 65: 1503-1505

Buccafusco JJ (1996) The role of central cholinergic neurons in the regulation of blood pressure and in experimental hypertension. Pharmacol Rev 48: 179-211.

Calvert CA, Wall TM (2001) Correlations among time and frequency measures of heart rate variability recorded by use of a Holter monitor in overtly healthy Doberman 
pinschers with and without echocardiographic evidence of dilated cardiomyopathy. Am J Vet Res 62: 1787-1792.

Eaton GM, Cody RJ, Nunziata E, Binkley PF (1995) Early left ventricular dysfunction elicits activation of sympathetic drive and attenuation of parasympathetic tone in paced canine model of congestive heart failure. Circulation 92: 555-561.

Eckberg DL, Drabinsky M, Braunwald E (1971) Defective cardiac parasympathetic control in patients with heart disease. N Engl J Med 285: 877-883.

Freeman LM, Rush JE, Farabaugh AE, Must A (2005) Development and evaluation of a questionnaire for assessing health-related quality of life in dogs with cardiac disease. J Am Vet Med Assoc 226: 1864-1868.

Gourine A, Bonder SI, Spyer KM, Gourine AV (2008) Beneficial effect of the central nervous system beta-adrenoceptor blockade on the failing heart. Circ Res 102: 633-636.

Häggström J, Hamlin RL, Hansson K, Kvart C (1996) Heart rate variability in relation to severity of mitral regurgitation in Cavalier King Charles spaniels. J Small Anim Pract 37: 69-75

Jurek S, Sredniawa B, Musialik-Łydka A, Kalarus Z (2007) Heart rate turbulence - a new predictor of sudden cardiac death. Polski Merkuriusz Lekarski 23: 55-59

Koyama J, Watanabe J, Yamada A, Koseki Y, Konno Y, Toda S, Shinozaki T, Miura M, Fukuchi M, Ninomiya M, Kagaya Y, Shirato K (2002) Evaluation of heart-rate turbulence as a new prognostic marker in patients with chronic heart failure. Circ J 66: 902-907.

Kubo T, Goshima Y, Hata H, Misu Y (1990) Evidence that endogenous catecholamines are involved in alpha 2-adrenoceptor-mediated modulation of the aortic baroreceptor reflex in the nucleus tractus solitarii of the rat. Brain Res 526: 313-317.

Lawrence AJ, Jarrott B (1996) Neurochemical modulation of cardiovascular control in the nucleus tractus solitarius. Prog Neurobiol 48: 21-53.

Leenen FH (2007) Brain mechanisms contributing to sympathetic hyperactivity and heart failure. Circ Res 101: 221-223.
Little CJ, Julu PO, Hansen S, Reid SW (1999) Real-time measurement of cardiac vagal tone in conscious dogs. Am J Physiol 276: H758-H765

Little CJ, Julu PO, Hansen S, Reid SW (2005) Non-invasive real-time measurements of cardiac vagal tone in dogs with cardiac disease. Vet Rec 156: 101-105

Malberg H, Bauernschmitt R, Meyerfeldt U, Schirdewan A, Wessel N (2003) Short-term heart rate turbulence analysis versus variability and baroreceptor sensitivity in patients with dilated cardiomyopathy. Z Kardiol 92: 547$-557$.

Malik M, Wichterle D, Schmidt G (1999) Heart rate turbulence. G Ital Cardiol 29: 65-69.

Marine JE, Watanabe MA, Smith TW, Monahan KM (2002) Effect of atropine on heart rate turbulence. Am J Cardiol 89: 767-769.

Noszczyk-Nowak A, Pasławska U, Nicpoń J (2009) ECG parameters in 24-hour Holter monitoring in healthy dogs. Bull Vet Inst Pulawy 53: 499-502.

Oyama MA, Chittur SV, Reynolds CA (2009) Decreased triadin and increased calstabin 2 expression in Great Danes with dilated cardiomyopathy. J Vet Intern Med 23: $1014-1019$

Sade E, Aytemir K, Oto A, Nazli N, Ozmen F, Ozkutlu H, Tokgözoglu L, Aksöyek S, Ovünç K, Kabakçi G, Ozer N, Kes S (2003) Assessment of heart rate turbulence in the acute phase of myocardial infarction for long-term prognosis. Pacing Clin Electrophysiol 26: 544-550.

Schmidt G, Malik M, Barthel P, Schneider R, Ulm K, Rolnitzky L, Camm AJ, Bigger JT Jr, Schömig A (1999) Heart-rate turbulence after ventricular premature beats as a predictor of mortality after acute myocardial infarction. Lancet 353: 1390-1396.

Wess G, Schulze A, Geraghty N, Hartmann K (2010) Ability of a 5-minute electrocardiography (ECG) for predicting arrhythmias in Doberman Pinschers with cardiomyopathy in comparison with a 24-hour ambulatory ECG. J Vet Intern Med 24: 367-371. 